Original Research Paper

\title{
Bayesian Approach to the Estimation of a Poisson Mean with Application to NBA Three Point Attempts
}

\author{
Patricia Pepple Williamson \\ Department of Mathematics, Applied Mathematics and Statistics, 10900 Euclid Avenue, \\ Case Western Reserve University, Cleveland, OH 44106-7058, 216-368-6013, USA
}

\author{
Article history \\ Received: 09-05-2018 \\ Revised: 23-07-2018 \\ Accepted: 08-11-2018 \\ Email: patricia.williamson@case.edu
}

\begin{abstract}
Bayesian Credible Intervals are proposed for a Poisson mean. These intervals are compared to five classical confidence intervals found in the literature. A simulation study is performed to compare the procedures using two different criteria and it is attempted to determine which of the procedures performs best for various values of the parameter and sample size. Estimation of the number of three-point shot attempts and three-point shot makes by the San Antonio Spurs is given as an example.
\end{abstract}

Keywords: Pivotal Quantity, Bayesian Credible Interval, Noninformative Prior, Posterior Distribution, Expected Fisher Information

\section{Introduction}

During the 2011-2012, 2012-2013 and 2013-2014 regular seasons, the San Antonio Spurs were in the top three in team three point shooting percentage. Because a random phenomenon for which a count of some type may be modeled by a Poisson distribution, the number of three-point attempts and the number of three pointers made in a game are possible candidates. Because it seems reasonable to assume that these two counts are such that at most one three point attempt (or one make) can occur in a very small interval of time and the number of attempts (or the number of makes) in two equal nonoverlapping time intervals should be independent and have the same distribution, the Poisson distribution seems like an appropriate model for each of these counts.

There have been many confidence intervals proposed and compared in the literature to estimate a Poisson mean. A sample of such work are Sahai and Khurshid (1993), Schwertman and Martinez (1994), Barker (2002), Byrne and Kabaila (2005), Patil and Kulkarni (2012), Khamkong (2012) and Tanusit (2012); most of these include the familiar Wald interval, the scores interval and the Garwood interval which are considered in this paper. For six approximate intervals and the exact Garwood interval, Schwertman and Martinez (1994) produces tables for a range of observed Poisson values and several confidence levels. Barker (2002) compares a total of nine confidence intervals using coverage rate, expected length and whether or not the interval is in closed form. Byrne and Kabaila (2005) compares at least twelve approximate and exact confidence intervals using coverage rate and length; one is a short exact interval and is calculated via an algorithm and was first proposed in Kabaila and Byrne (2001). Patil and Kulkarni (2012) compares 19 confidence intervals using several criteria including coverage rate and expected length. Sahai and Khurshid (1993) gives several nice biomedical and epidemiological examples which could be modeled by the Poisson distribution. Also, they provide a review of the methodology for ten confidence intervals; each of these intervals are in the comparative study of Patil and Kulkarni (2012) including the Wald, scores and Garwood intervals. Tanusit (2012) compares seven confidence intervals using coverage rate and length; three of which adapt three common intervals replacing the typical point estimator of the Poisson mean (the sample mean) by a Bayes estimator of a Poisson mean utilizing the conjugate Gamma prior with criterion given for the choice of the prior parameters. Khamkong (2012) compares four confidence intervals using coverage rate and estimated expected length; the scores interval is considered along with a proposed adapted Wald interval which outperformed the other intervals for small mean and small to moderate sample sizes.

Assuming $X$ has a Poisson distribution with mean $\theta$ (Poisson $(\theta))$, note that $X$ has an approximate normal distribution with $\mu=\theta=\sigma^{2}(\mathrm{~N}(\theta, \theta))$ for large $\theta$. This can be seen by assuming $X_{1}, \ldots, X_{\theta}$ is a random sample from a Poisson (1) distribution, letting $X=\sum_{i=1}^{\theta} X_{i}$ and applying the Central Limit Theorem. In fact, for values of $\theta$ as small as 25 , the normal approximation works quite well. 
When $\theta$ is not small, assuming $X_{1}, \ldots, X_{n}$ is a random sample from a Poisson $(\theta)$ distribution is basically equivalent to assuming a random sample of size $n$ from a $\mathrm{N}(\theta, \theta)$ distribution. Estimating $\theta$ assuming a random sample of size $n$ from a $\mathrm{N}(\theta, \theta)$ distribution is an interesting problem in itself but is even more attractive with its application in estimating a Poisson mean. In interval estimation of $\theta$ assuming normality, a chi-square pivot $\left(Q_{1}=(n-1) S^{2} / \theta\right)$, a t pivot $\left(Q_{2}=\sqrt{n}(\bar{X}-\theta) / S\right)$ and a $\mathrm{N}(0,1)$ pivot $\left(Q_{3}=\sqrt{n}(\bar{X}-\theta) / \sqrt{\theta}\right)$, where $\bar{X}=(1 / n) \sum_{i=1}^{n} X_{\mathrm{i}}$ and $S^{2}=\sum_{i=1}^{n}\left(X_{i}-\bar{X}\right)^{2} /(n-1)$, may be employed. Also, $Q_{4}=\sqrt{n}(\bar{X}-\theta) / \sqrt{\bar{X}}$ is a reasonable pivot which is approximately $\mathrm{N}(0,1)$ for large $n$ when sampling from a Poisson $(\theta)$ distribution. Additionally, Garwood (1936) gives an exact interval for the Poisson mean which is valid for any value of $n$.

As alternatives to the classical intervals indicated above, two Bayesian credible intervals are proposed. The first approach utilizes the Jeffrey's prior assuming a $\mathrm{N}(\theta, \theta)$ distribution and the second approach employs the Jeffrey's prior assuming a Poisson $(\theta)$ distribution.

In section 2, the classical confidence intervals are given using pivots $Q_{1}, Q_{2}, Q_{3}$ and $Q_{4}$ along with the exact confidence interval first derived by Garwood (1936). The two new Bayesian credible intervals are derived in section 3. Section 4 modifies the previous interval estimators of $\theta$ based on a single Poisson $(\theta)$ random variable when $\theta$ is large. A simulation study is performed to compare the procedures using two different criteria in section 5 . The three pointer example is given in section 6 along with a summary of results to conclude the paper. It will be apparent from the numerical studies which of the procedures performs best for various values of $\theta$ and $n$.

\section{Confidence Intervals Using the Pivotal Quantity Method}

Let $X=\left(X_{1}, \ldots, X_{n}\right)$ be a random sample and let $T_{1}=$ $\mathrm{t}_{1}(X)$ and $T_{2}=t_{2}(X)$ be two statistics satisfying $T_{1} \leq T_{2}$ and $P_{\theta}\left[T_{1}<\theta<T_{2}\right]=1-\alpha$, where $\alpha$ is between 0 and 1 and does not depend on $\theta$, then the interval $\left(T_{1}, T_{2}\right)$ is called a $100(1-\alpha) \%$ confidence interval for $\theta$. A pivotal quantity $Q$ is a function of $X$ and $\theta$ which has a distribution free of $\theta$. To find a $100(1-\alpha) \%$ confidence interval for $\theta$, find $q_{1}$ and $q_{2}$ such that $\mathrm{P}\left[q_{1}<Q<q_{2}\right]=1$ $\alpha$. The values $q_{1}$ and $q_{2}$ will depend on $\alpha$ and $X$. Manipulate $\left\{q_{1}<Q<q_{2}\right\}$ so that $\left\{t_{1}(X)<\theta<t_{2}(X)\right\}$, then $\left(T_{1}, T_{2}\right)$ is a $100(1-\alpha) \%$ confidence interval for $\theta$ where $T_{i}=t_{i}(X)$ for $i=1$ and 2 .

Assuming $X_{1}, \ldots, X_{n}$ is a random sample from a $\mathrm{N}(\theta, \theta)$ distribution, the pivotal quantity $Q_{1}=(n-1) S^{2} / \theta$ has a chi-square distribution with $(n-1)$ degrees of freedom $\left(\chi^{2}(n-1)\right)$. Letting $\mathrm{q}_{1}=\chi_{1-\alpha / 2, n-1}^{2}$ and $q_{2}=\chi_{\alpha / 2, n-1}^{2}$, where $\chi_{\gamma, k}^{2}$ denotes the $100(1-\gamma)$ th percentile of a $\chi^{2}(k)$ distribution, note that $P\left[\chi_{1-\alpha / 2, n-1}^{2}<Q_{1}<\chi_{\alpha / 2, n-1}^{2}\right]=1-\alpha$. Manipulating:

$$
\left\{\chi_{1-a / 2, n-1}^{2}<(n-1) S^{2} / \theta<\chi_{a / 2, n-1}^{2}\right\}
$$

to isolate $\theta$, it is straightforward to show that a $100(1-$ $\alpha) \%$ confidence interval for $\theta$ is:

$\left((n-1) S^{2} / \chi_{\alpha / 2, n-1}^{2},(n-1) S^{2} / \chi_{1-a / 2, n-1}^{2}\right)$.

Assuming normality, the pivotal quantity $Q_{2}=$ $\sqrt{n}(\bar{X}-\theta) / S$ has a t-distribution with $(n-1)$ degrees of freedom $(t(n-1))$. Letting $t_{\alpha / 2, n-1}$ denote the 100(1$\alpha / 2)$ th percentile of a $t(n-1)$ distribution and noting that $\mathrm{P}\left[-t_{\alpha / 2, n-1}<Q_{2}<t_{\alpha / 2, n-1}\right]=1-\alpha$, the resulting $100(1-$ $\alpha) \%$ confidence interval for $\theta$ is given by:

$$
\bar{X} \pm \mathrm{t}_{\alpha / 2, n-1} S / \sqrt{n} .
$$

The statistic $Q_{3}=\sqrt{n}(\bar{X}-\theta) / \sqrt{\theta}$ is a pivotal quantity because it is a function of $X$ and it has a $\mathrm{N}(0,1)$ distribution which is free of $\theta$. Let $q_{1}=-z_{\alpha / 2}$ and $q_{2}=$ $z_{\alpha / 2}$, where $z_{\alpha / 2}$ denotes the $100(1-\alpha / 2)$ th percentile of a standard normal distribution. Employing the pivotal quantity method, the resulting $100(1-\alpha) \%$ confidence interval for $\theta$ is given by:

$$
\bar{X}+\mathrm{z}_{\alpha / 2}^{2} /(2 n) \pm z_{\alpha / 2} \sqrt{\bar{X} / n+\mathrm{z}_{\alpha / 2}^{2} /\left(4 n^{2}\right)} .
$$

This result is sometimes referred to as the scores interval.

When $X_{1}, \ldots, X_{n}$ is a random sample from a Poisson $(\theta)$ distribution, $Q_{4}=\sqrt{n}(\bar{X}-\theta) / \sqrt{\bar{X}}$ is approximately $\mathrm{N}(0,1)$ for large $n$. Using this pivotal quantity, it follows that:

$$
\bar{X} \pm \mathrm{z}_{\alpha / 2} \sqrt{\bar{X} / n}
$$

is an approximate $100(1-\alpha) \%$ confidence interval for $\theta$ which is the familiar Wald interval.

To find the exact Garwood interval, let $W=$ $\sum_{i=1}^{n} X_{\mathrm{i}}$ assuming a Poisson $(\theta)$ random sample. Noting that $W$ is Poisson $(n \theta)$ and observing $W=w$, it is desired to find $\theta$ such that $\sum_{k=0}^{w} \exp (-n \theta)(n \theta)^{\mathrm{k}} / k !=\alpha / 2$ and $\sum_{k=\mathrm{w}}^{\infty} \exp (-n \theta)(n \theta)^{\mathrm{k}} / k !=\alpha / 2$. A helpful result that appears in 
many mathematical statistics textbooks (e.g., Mood et al. (1974)) that relates the Poisson and Gamma families is:

$$
\begin{aligned}
& P(U \leq u)=1-\sum_{k=0}^{r-1} \exp (-u \theta)(u \theta)^{\mathrm{k}} / k ! \\
& =\sum_{k=r}^{\infty} \exp (-u \theta)(u \theta)^{\mathrm{k}} / k !
\end{aligned}
$$

where, $U$ has a $\operatorname{Gamma}(r, \theta)$ distribution. To satisfy $\sum_{k=\mathrm{w}}^{\infty} \exp (-n \theta)(n \theta)^{\mathrm{k}} / k !=\alpha / 2$, it is necessary that $\alpha / 2=\mathrm{P}(U$ $\leq n)=\mathrm{P}(V \leq 2 \theta n)$ using $(5)$, where $U$ has a $\operatorname{Gamma}(w, \theta)$ distribution and $V=2 \theta U$ has a chi-square distribution with $2 w$ degrees of freedom $\left(\chi^{2}(2 w)\right)$. Hence, $\theta=$ $\chi_{1-\alpha / 2,2 w}^{2} /(2 n)$. Similarly, for $\sum_{k=0}^{w} \exp (-n \theta)(n \theta)^{\mathrm{k}} / k !=\alpha / 2$ to be satisfied, it is necessary that:

$$
\begin{aligned}
& \alpha / 2=1-\sum_{k=w+1}^{\infty} \exp (-n \theta)(n \theta)^{\mathrm{k}} / k ! \\
& =1-P(U \leq n)=P(U>n)=P(V>2 \theta n)
\end{aligned}
$$

using (5), where $U$ has a $\operatorname{Gamma}(w+1, \theta)$ distribution and $V=2 \theta U$ has a $\chi^{2}(2(w+1))$ distribution. It follows that $\theta=\chi_{a / 2,2(w+1)}^{2} /(2 n)$ and the resulting Garwood 100(1$\alpha) \%$ confidence interval for $\theta$ is:

$$
\left(\chi_{1-\alpha / 2,2 w}^{2} /(2 n), \chi_{a / 2,2(w+1)}^{2} /(2 n)\right) .
$$

Although the exact method of Barker (2002) was to find the smallest $\theta$ such that $\sum_{k=0}^{w} \exp (-n \theta)(n \theta)^{\mathrm{k}} / k ! \leq$ $\alpha / 2$ and the largest $\theta$ such that $\sum_{k=\mathrm{w}}^{\infty} \exp (-n \theta)(n \theta)^{\mathrm{k}} / k ! \leq$ $\alpha / 2$ which produce a confidence interval not in closed form, he did not use the result in (5) to derive (6) and did not refer to this interval as the Garwood interval. However, Sahai and Khurshid (1993) briefly gives this argument for $n=1$.

\section{Bayesian Credible Intervals}

A $100(1-\alpha) \%$ Bayesian credible interval for $\theta$ is $\left(t_{1}(x), t_{2}(x)\right)$, where $x$ is an observed value of $X=\left(X_{1}, \ldots\right.$, $\left.X_{n}\right), \mathrm{t}_{1}(x) \leq t_{2}(x)$ and $P\left[t_{1}(x) \leq \theta \leq t_{2}(x) \mid x\right]=1-\alpha$. This probability is with respect to the posterior distribution of $\theta$ given $x$ which is given by:

$$
\pi(\theta \mid x)=f(x \mid \theta) \pi(\theta) / m(x)
$$

where, $f(x \mid \theta)$ is the joint density of $X, \pi(\theta)$ is the prior density of $\theta$ and $m(x)$ is the marginal density of $X$. In
Bayesian analysis, all inference is based on the posterior distribution.

Assuming $X_{1}, \ldots, X_{n}$ is a random sample from a normal distribution with mean and variance equal to $\theta$, the joint probability density function of $X_{1}, \ldots, X_{n}$ is:

$$
f(x \mid \theta)=\prod_{i} f\left(x_{i} \mid \theta\right)=(2 \pi \theta)^{-n / 2} \exp \left\{-\sum_{i=1}^{n}\left(x_{i}-\theta\right)^{2} /(2 \theta)\right\} .
$$

Assuming no prior knowledge is available for the parameter $\theta$, a noninformative prior is employed using the method of Jeffreys (1961). This method defines the noninformative prior of $\theta$ to be $\pi(\theta)=[I(\theta)]^{1 / 2}$, where $I(\theta)$ is the expected Fisher information given by:

$$
I(\theta)=-E\left[\frac{\partial^{2} \ln \left(f\left(X_{i} \mid \theta\right)\right)}{\partial \theta^{2}}\right]
$$

Because $\ln \left(f\left(x_{i} \mid \theta\right)\right)=-(1 / 2) \ln (2 \pi)-(1 / 2) \ln (\theta)-\left(x_{i^{-}}\right.$ $\theta)^{2} /(2 \theta)$ :

$$
\frac{\partial^{2} \ln \left(f\left(x_{i} \mid \theta\right)\right)}{\partial \theta^{2}}=1 /\left(2 \theta^{2}\right)-x_{i}^{2} / \theta^{3} .
$$

It follows that $I(\theta)=\mathrm{E}\left[X_{i}^{2} / \theta^{3}-1 /\left(2 \theta^{2}\right)\right]=1 / \theta+1 /\left(2 \theta^{2}\right)$ and hence:

$$
\pi(\theta)=\left[1 / \theta+1 /\left(2 \theta^{2}\right)\right]^{1 / 2}
$$

From (7), the resulting posterior density of $\theta$ given $x$ is:

$$
\pi(\theta \mid x) \propto \theta^{-n / 2} \exp \left\{-\sum_{i=1}^{n} x_{i}^{2} /(2 \theta)-n \theta / 2\right\}\left[1 / \theta+1 /\left(2 \theta^{2}\right)\right]^{1 / 2} .
$$

Figure 1 plots $\pi(\theta \mid x)$ where $x_{1}, \ldots, x_{n}$ were randomly generated from a $\mathrm{N}(\theta, \theta)$ distribution with $n=20$ and $\theta=$ 100; the posterior looks fairly normal. For this particular $x$ generated $(x=(101.32039,98.72714,93.60091$, 88.72688, 111.43617, 103.49016, 109.97538, 104.67434, 120.22155, 105.63300, 90.54763, 104.42001, 95.76609, 110.46566, 104.04043, 101.49670, 102.85183, 108.18623, $102.35371,99.56476)$ ), the posterior mean and variance were 102.68 and 5.1116, respectively.

Assuming the posterior distribution of $\theta$ is approximately normal with mean $\hat{\mu}_{N}=E(\theta \mid x)$ and variance $\hat{\sigma}_{N}^{2}=\operatorname{Var}(\theta \mid x)$, an approximate $100(1-\alpha) \%$ Bayesian credible interval for $\theta$ is:

$\hat{\mu}_{N} \pm z_{\alpha / 2} \hat{\sigma}_{N}$ 


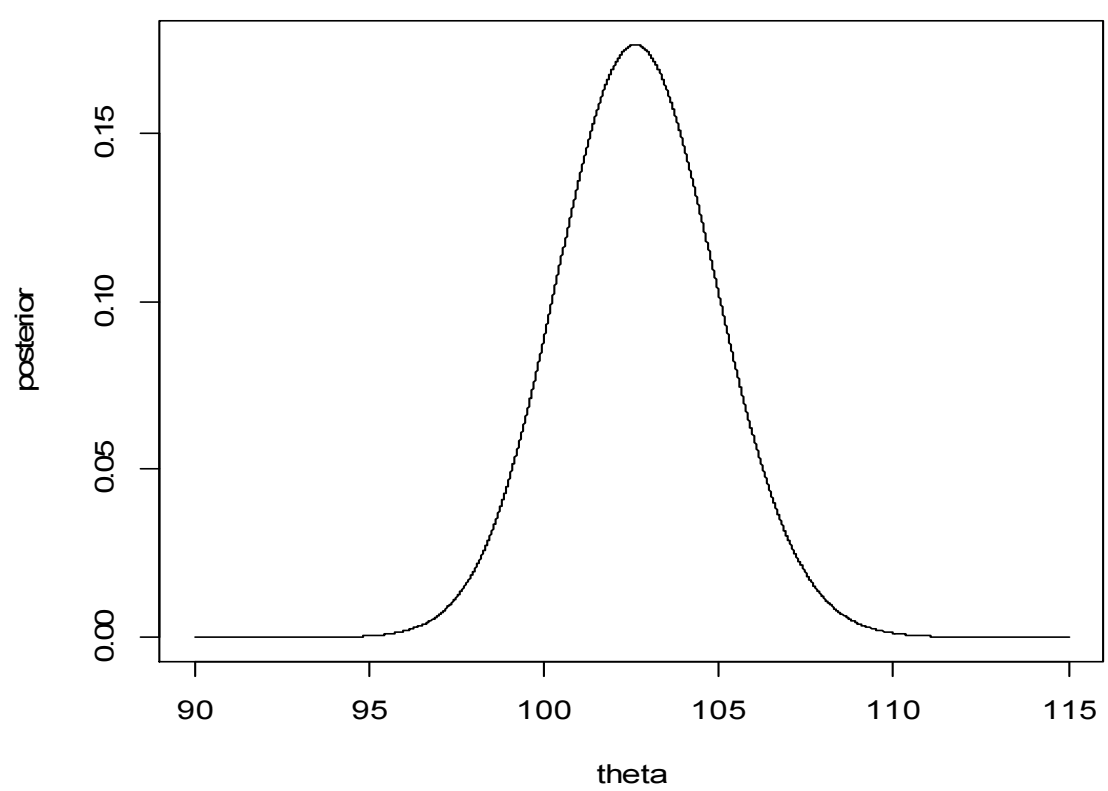

Fig. 1: Posterior density of $\theta$ given $x$ where $n=20$ and $\theta=100$

Note for the sample that generated Fig. 1, the 95\% credible interval for $\theta$ given in (9) is $(98.25,107.11)$ which contains 100 .

Now assume $X_{1}, \ldots, X_{n}$ is a random sample from a Poisson distribution with mean $\theta$; hence, the joint probability mass function of $X_{1}, \ldots, X_{n}$ is:

$$
f(x \mid \theta)=\theta^{\sum_{i=1}^{n} x_{i}} \exp (-n \theta) / \prod_{i=1}^{n} x_{i} !
$$

Employing Jeffreys' method again, first note that $\ln \left(f\left(x_{i} \mid \theta\right)\right)=x_{i} \ln (\theta)-\theta-\ln \left(x_{i} !\right)$. Hence:

$$
I(\theta)=-E\left[\frac{\partial^{2} \ln \left(f\left(X_{i} \mid \theta\right)\right)}{\partial \theta^{2}}\right]=E\left(X_{i} / \theta^{2}\right)=1 / \theta
$$

this yields the noninformative prior $\pi(\theta)=\theta^{-1 / 2}$. Using (10), the resulting posterior density of $\theta$ given $x$ is thus given by:

$\pi(\theta \mid x) \propto \theta^{\sum_{i=1}^{n} x_{i}-1 / 2} \exp (-n \theta)$.

Figure 2 plots the posterior in (11) where $x_{1}, \ldots, x_{50}$ were randomly generated from a Poisson(100) distribution. This posterior looks fairly normal with posterior mean and variance given by 98.99 and 1.9798 , respectively.

Assuming the posterior distribution of $\theta$ is approximately normal with mean $\hat{\mu}_{P}=E(\theta \mid x)$ and variance $\hat{\sigma}_{P}^{2}=\operatorname{Var}(\theta \mid x)$, an approximate $100(1-\alpha) \%$ Bayesian credible interval for $\theta$ is:

$\hat{\mu}_{P} \pm z_{\alpha / 2} \hat{\sigma}_{P}$

For the sample that generated Fig. 2, the 95\% credible interval for $\theta$ given in (12) is $(96.23,101.75)$ which contains 100 .

\section{Modification of Intervals Assuming a Single Poisson Random Variable}

Consider modifying the previous interval estimators of $\theta$ in Sections 2 and 3 based on a single Poisson $(\theta)$ random variable when $\theta$ is large. Letting $Y$ have a $\operatorname{Poisson}(\theta)$ distribution, the previous confidence intervals and credible intervals are modified using $n=1$. First note that the chisquare confidence interval in (1) and the t interval in (2) cannot be altered because the observed value of the standard deviation is 0 in this case due to $n$ being 1 . However, the remaining intervals can be modified.

The scores confidence interval based on $Q_{3}$ given in (3) becomes:

$Y+\mathrm{z}_{\alpha / 2}^{2} / 2 \pm z_{\alpha / 2} \sqrt{Y+\mathrm{z}_{\alpha / 2}^{2} / 4}$

The Wald confidence interval based on $Q_{4}$ given in (4) modified for a single $\operatorname{Poisson}(\theta)$ random variable is given by:

$Y \pm \mathrm{z}_{\alpha / 2} \sqrt{Y}$ 


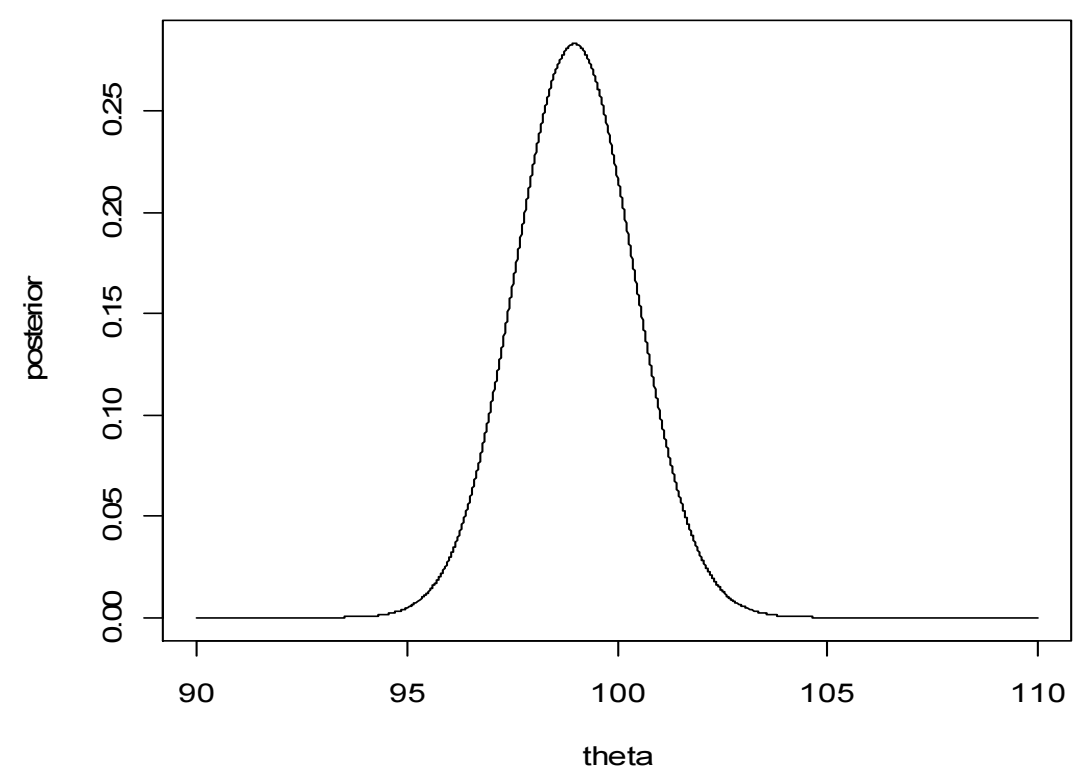

Fig. 2: Posterior density of $\theta$ given $x$ where $n=50$ and $\theta=100$

Observing $Y=y$, the resulting Garwood 100(1- $\alpha) \%$ confidence interval for $\theta$ is:

$$
\left(\chi_{1-\alpha / 2,2 y}^{2} / 2, \chi_{\alpha / 2,2(y+1)}^{2} / 2\right) \text {. }
$$

To derive the Bayesian credible intervals, note from (8) that the posterior of $\theta$ given $y$ in the normal case is:

$$
\pi(\theta \mid y) \propto \theta^{-1 / 2} \exp \left\{-y^{2} /(2 \theta)-\theta / 2\right\}\left[1 / \theta+1 /\left(2 \theta^{2}\right)\right]^{1 / 2} .
$$

Letting $\hat{\mu}_{N}$ and $\hat{\sigma}_{N}^{2}$ denote the mean and variance of the posterior given in (16), respectively and assuming this posterior is close to normal, an approximate $100(1-\alpha) \%$ Bayesian credible interval for $\theta$ is as given in (9) using the new definitions of $\hat{\mu}_{N}$ and $\hat{\sigma}_{N}$. Similarly for the Poisson case, the posterior density of $\theta$ given $y$ is given by:

$$
\pi(\theta \mid y) \propto \theta^{y-1 / 2} \mathrm{e}^{-\theta} .
$$

Assuming this posterior is approximately normal with $\hat{\mu}_{P}=E(\theta \mid y)$ and variance $\hat{\sigma}_{P}^{2}=\operatorname{Var}(\theta \mid y)$, an approximate $100(1-\alpha) \%$ Bayesian credible interval for $\theta$ is as given in (12) using the new definitions of $\hat{\mu}_{p}$ and $\hat{\sigma}_{p}$.

Figure 3 plots the posterior densities given in (16) and (17) where a variate of 103 was randomly generated from a Poisson distribution with $\theta=100$. These posteriors look fairly normal, where $\hat{\mu}_{N}=103.4933, \hat{\sigma}_{N}^{2}$ $=103.8477, \hat{\mu}_{P}=103.4988$ and $\hat{\sigma}_{P}^{2}=103.4409$. These densities are nearly indistinguishable. For $y=103$, the $95 \%$ credible intervals given in (9) and (12) using the new definitions of $\hat{\mu}_{N}, \hat{\sigma}_{N}, \hat{\mu}_{P}$ and $\hat{\sigma}_{P}$ are (83.52, $123.47)$ and $(83.56,123.43)$, respectively. Both of the intervals contain 100, the assumed value of $\theta$ and the second interval based on the posterior in (17) is slightly narrower than the one based on the posterior in (16).

\section{Numerical Studies}

To compare the seven approaches, a simulation study is performed considering various values of the parameter $\theta$ and the sample size $n$ for the two different underlying distributions from which the random sample is taken. Approaches 1 through 4 utilize the confidence intervals for $\theta$ given in (1), (2), (3) and (4), respectively, approaches 5 and 6 utilize the credible intervals for $\theta$ given in (9) and (12), respectively and approach 7 is the Garwood confidence interval in (6). Approaches 1 and 2 do not exist for $n=1$ because the sample variance equals 0 . For $n=1$, approaches 3, 4 and 7 employ the confidence intervals given in (13), (14) and (15), respectively and approaches 5 and 6 use the intervals given in (9) and (12) employing the posterior densities given in (16) and (17), respectively.

All combinations with $n$ equal to $5,10,20,50$ and 100 and the parameter $\theta$ equal to $1,5,10,20,50$ and 100 assuming the $\mathrm{N}(\theta, \theta)$ and $\operatorname{Poisson}(\theta)$ distributions are considered. Also, values of $\theta$ equal to 50, 100, 150, 200, $250,300,350$ and 400 for $n=1$ assuming a Poisson( $\theta$ ) distribution are investigated. For each $n, \theta$ and distribution combination, a random sample is generated and the various intervals are computed, where it is noted whether or not $\theta$ is in each of the resulting intervals as well as the length of each of the resulting intervals. 


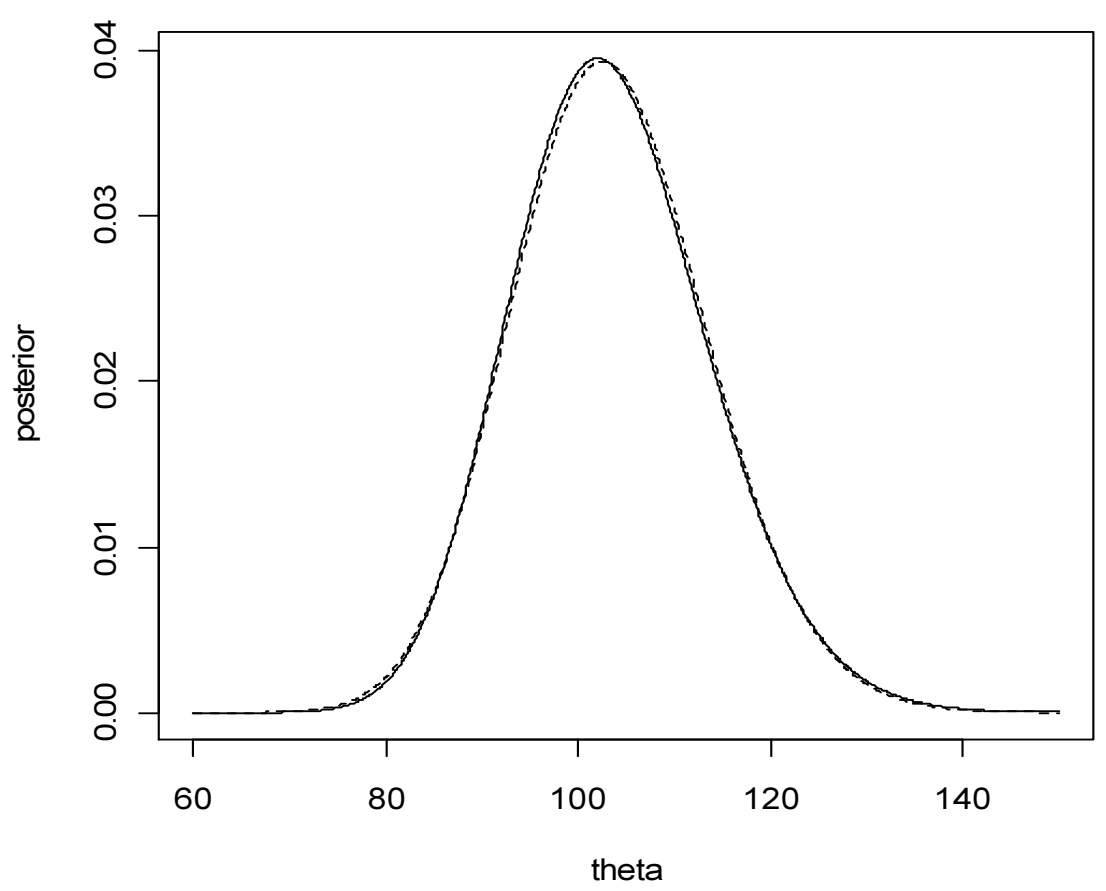

Fig. 3: Posterior densities of $\theta$ given $y=103$ where $\theta=100$ given in (16) and (17) (dashed line)

This process is repeated 10,000 times and the percentage of time the respective interval estimates cover the parameter and the average lengths of the respective interval estimates are calculated. Casella and Berger (2002) suggest size and coverage probability as criteria to evaluate confidence intervals. Bolded values in the table indicate the approach that yields the smallest average length among all approaches with coverage percentages of at least $95 \%$. Italic bolded values signify the approach that yields the smallest average length among all approaches with coverage percentages of at least $94.50 \%$ which could be rounded to $95 \%$.

Recall that the criteria of coverage percentage and length closely match the criteria of Barker (2002) which also included the criterion of the interval being in closed form. The Wald interval, the scores interval, the Garwood interval and six others for values of $n \theta$ between 0.5 and 5.0 were compared using these three criteria. Noting that Barker did not point out that the method that was referred to as the exact method was actually the method of Garwood which yields an interval of closed form, Barker recommends the Garwood interval when desiring the coverage percentage to never go below $95 \%$ and the scores interval when tolerating approximate coverage percentage and wide intervals. In the numerical study of Barker, the Garwood interval was always narrower than the scores interval. The opposite is the case in this numerical study; however, the numerical study of Barker considers values of $n \theta$ not exceeding 5 . Note also that the Wald interval performed poorly in terms of coverage in the Barker numerical study.
Sampling from a normal distribution, Table 1 gives the results for the various $\theta$ and $n$ when $n>1$. First note that the coverage percentages of all of the approaches are all around 95\% for most of the values of $\theta$ and $n$ considered. The only exceptions to this are approaches 4 (Wald) and 6 (second Bayes) which have low coverage percentages for small $\theta$ and $n$ and approach 7 (Garwood) which has high coverage percentages for small $\theta$ and $n$. It is not surprising that approach 4 does poorly for small $n$ as it is based on $Q_{4}$ which is approximately standard normal for large $n$; however, even for small $n$, approach 4 has good coverage percentages for larger $\theta$. Note also that approach 4 cannot be used when $\bar{x}$ is negative. This happened in the simulation study only when $\theta=1$ and $n$ $=5$ or 10 ; in such cases, the coverage percentages and average lengths were based on the number of loops where $\bar{x}$ was positive which was over $99 \%$ of the time. Similarly, approach 3 (scores) cannot be used when the radicand in (3) is negative. This occurred in only a few loops in the simulations when $\theta=1$ and $n=5$ or 10 and adjustments were accordingly made. The Bayesian approach assuming normal data (approach 5) tended to yield the smallest average length while keeping coverage probabilities of at least 0.95 ; the only exception to this was when $n=5$ for the various $\theta$ where approach 5 had the smallest average length but had coverage percentages slightly below $95 \%$. Except for the $\theta=1$ or $n=5$ cases, approach 4 (Wald) performed well followed by approaches 6 (second Bayes) and 3 (scores). When $n=5$, approach 3 performed best for smaller values of $\theta$. For 
any $\theta$, approaches 3,4 and 6 have very close average lengths for $n=50$ and 100; however, approach 5 has smallest average lengths in these cases. Approach 7 (Garwood) outperformed the others in only one case $(\theta=$ $50, n=5$ ) where several others had smaller average lengths but had coverage percentages a little below $95 \%$. This is not surprising as this interval is not based on normal data. Approach 1 is the worst as its average length far exceeds the other intervals while having reasonable coverage percentages.

Table 1: Coverage percentages and average length of interval estimates for $\theta$ for the seven approaches for various $\theta, n$ and underlying normal distribution $(\mathrm{N}(\theta, \theta))$

\begin{tabular}{|c|c|c|c|c|c|c|c|}
\hline & \multicolumn{7}{|c|}{ Approach } \\
\hline & 1 & 2 & 3 & 4 & 5 & 6 & 7 \\
\hline \multicolumn{8}{|l|}{$\theta=1, n=5$} \\
\hline Coverage $\%$ & 95.29 & 95.11 & 95.29 & 91.30 & 94.78 & 93.23 & 97.75 \\
\hline $\begin{array}{l}\text { Average length } \\
\theta=1, n=10\end{array}$ & 7.880 & 2.000 & 1.884 & 1.695 & 1.597 & 1.755 & 1.986 \\
\hline Coverage \% & 95.14 & 95.08 & 94.94 & 92.91 & 95.32 & 94.28 & 96.79 \\
\hline $\begin{array}{l}\text { Average length } \\
\theta=1, n=20\end{array}$ & 2.877 & 1.359 & 1.286 & 1.226 & 1.078 & 1.257 & 1.349 \\
\hline Coverage $\%$ & 94.85 & 95.34 & 95.30 & 93.88 & 95.18 & 94.68 & 96.18 \\
\hline $\begin{array}{l}\text { Average length } \\
\theta=1, n=50\end{array}$ & 1.556 & 0.923 & 0.892 & 0.871 & 0.738 & 0.882 & 0.927 \\
\hline Coverage \% & 95.37 & 95.40 & 95.38 & 94.50 & 95.17 & 95.02 & 95.64 \\
\hline $\begin{array}{l}\text { Average length } \\
\theta=1, n=100\end{array}$ & 0.857 & 0.566 & 0.558 & 0.553 & 0.459 & 0.556 & 0.575 \\
\hline Coverage \% & 95.05 & 95.33 & 95.31 & 95.04 & 95.15 & 95.04 & 95.79 \\
\hline $\begin{array}{l}\text { Average length } \\
\theta=5, n=5\end{array}$ & 0.577 & 0.395 & 0.394 & 0.392 & 0.322 & 0.393 & 0.402 \\
\hline Coverage \% & 94.88 & 94.92 & 95.19 & 93.95 & 94.54 & 93.92 & 96.08 \\
\hline $\begin{array}{l}\text { Average length } \\
\theta=5, n=10\end{array}$ & 39.502 & 5.189 & 3.979 & 3.903 & 3.722 & 3.814 & 4.022 \\
\hline Coverage \% & 94.72 & 95.15 & 95.40 & 94.56 & 95.15 & 94.94 & 95.96 \\
\hline $\begin{array}{l}\text { Average length } \\
\theta=5, n=20\end{array}$ & 14.243 & 3.104 & 2.790 & 2.763 & 2.672 & 2.774 & 2.871 \\
\hline Coverage $\%$ & 94.96 & 95.04 & 95.13 & 95.01 & 95.16 & 95.24 & 95.72 \\
\hline $\begin{array}{l}\text { Average length } \\
\theta=5, n=50\end{array}$ & 7.753 & 2.062 & 1.966 & 1.957 & 1.880 & 1.962 & 2.011 \\
\hline Coverage \% & 95.01 & 94.95 & 95.18 & 95.05 & 95.03 & 95.16 & 95.56 \\
\hline $\begin{array}{l}\text { Average length } \\
\theta=5, n=100\end{array}$ & 4.269 & 1.263 & 1.241 & 1.239 & 1.185 & 1.240 & 1.260 \\
\hline Coverage $\%$ & 95.06 & 95.19 & 95.16 & 95.10 & 95.35 & 95.18 & 95.44 \\
\hline $\begin{array}{l}\text { Average length } \\
\theta=10, n=5\end{array}$ & 2.895 & 0.885 & 0.877 & 0.876 & 0.837 & 0.877 & 0.887 \\
\hline Coverage $\%$ & 95.10 & 95.29 & 95.24 & 94.64 & 95.03 & 94.99 & 95.98 \\
\hline $\begin{array}{l}\text { Average length } \\
\theta=10, n=10\end{array}$ & 79.200 & 7.384 & 5.579 & 5.526 & 5.328 & 5.532 & 5.745 \\
\hline Coverage $\%$ & 95.11 & 95.19 & 95.23 & 95.14 & 95.33 & 95.29 & 95.84 \\
\hline $\begin{array}{l}\text { Average length } \\
\theta=10, n=20\end{array}$ & 28.766 & 4.415 & 3.934 & 3.915 & 3.850 & 3.925 & 4.022 \\
\hline Coverage $\%$ & 95.19 & 95.37 & 95.04 & 95.06 & 95.03 & 95.21 & 95.41 \\
\hline $\begin{array}{l}\text { Average length } \\
\theta=10, n=50\end{array}$ & 15.628 & 2.929 & 2.778 & 2.771 & 2.715 & 2.775 & 2.824 \\
\hline Coverage $\%$ & 95.03 & 95.32 & 95.15 & 95.02 & 95.18 & 95.04 & 95.26 \\
\hline $\begin{array}{l}\text { Average length } \\
\theta=10, n=100\end{array}$ & 8.532 & 1.786 & 1.754 & 1.753 & 1.713 & 1.753 & 1.773 \\
\hline Coverage $\%$ & 95.04 & 95.22 & 95.35 & 95.25 & 95.07 & 95.23 & 95.35 \\
\hline $\begin{array}{l}\text { Average length } \\
\theta=20, n=5\end{array}$ & 5.792 & 1.252 & 1.240 & 1.239 & 1.211 & 1.240 & 1.249 \\
\hline Coverage $\%$ & 95.30 & 95.27 & 95.15 & 95.01 & 95.09 & 95.18 & 95.68 \\
\hline Average length & 156.05 & 10.382 & 7.865 & 7.828 & 7.766 & 7.823 & 8.040 \\
\hline
\end{tabular}


Table 1: Continued

\begin{tabular}{|c|c|c|c|c|c|c|c|}
\hline & 1 & 2 & 3 & 4 & 5 & 6 & 7 \\
\hline \multicolumn{8}{|l|}{$\theta=20, n=10$} \\
\hline Coverage $\%$ & 94.89 & 95.17 & 95.21 & 95.09 & 95.13 & 95.17 & 95.68 \\
\hline $\begin{array}{l}\text { Average length } \\
\theta=20, n=20\end{array}$ & 57.420 & 6.236 & 5.556 & 5.543 & 5.492 & 5.546 & 5.648 \\
\hline Coverage $\%$ & 95.20 & 95.16 & 95.10 & 95.06 & 95.00 & 95.11 & 95.32 \\
\hline $\begin{array}{l}\text { Average length } \\
\theta=20, n=50\end{array}$ & 31.009 & 4.127 & 3.922 & 3.918 & 3.877 & 3.920 & 3.971 \\
\hline Coverage \% & 95.01 & 95.17 & 95.27 & 95.40 & 95.20 & 95.42 & 95.46 \\
\hline $\begin{array}{l}\text { Average length } \\
\theta=20, n=100\end{array}$ & 17.146 & 2.532 & 2.481 & 2.480 & 2.451 & 2.480 & 2.500 \\
\hline Coverage $\%$ & 95.19 & 95.13 & 95.17 & 95.22 & 95.14 & 95.22 & 95.32 \\
\hline $\begin{array}{l}\text { Average length } \\
\theta=50, n=5\end{array}$ & 11.563 & 1.770 & 1.753 & 1.753 & 1.732 & 1.753 & 1.763 \\
\hline Coverage $\%$ & 95.24 & 95.07 & 94.76 & 94.57 & 94.54 & 94.53 & 95.10 \\
\hline $\begin{array}{l}\text { Average length } \\
\theta=50, n=10\end{array}$ & 391.05 & 16.430 & 12.419 & 12.395 & 12.298 & 12.334 & 12.603 \\
\hline Coverage \% & 95.07 & 95.33 & 95.39 & 95.36 & 95.45 & 95.38 & 95.63 \\
\hline $\begin{array}{l}\text { Average length } \\
\theta=50, n=20\end{array}$ & 143.42 & 9.858 & 8.769 & 8.760 & 8.726 & 8.760 & 8.863 \\
\hline Coverage $\%$ & 95.04 & 95.48 & 95.20 & 95.16 & 95.22 & 95.23 & 95.37 \\
\hline $\begin{array}{l}\text { Average length } \\
\theta=50, n=50\end{array}$ & 77.878 & 6.539 & 6.200 & 6.197 & 6.171 & 6.199 & 6.248 \\
\hline Coverage \% & 95.15 & 95.35 & 95.36 & 95.32 & 95.36 & 95.34 & 95.30 \\
\hline $\begin{array}{l}\text { Average length } \\
\theta=50, n=100\end{array}$ & 42.839 & 4.003 & 3.920 & 3.920 & 3.901 & 3.920 & 3.941 \\
\hline Coverage $\%$ & 95.23 & 95.24 & 95.10 & 95.11 & 95.17 & 95.08 & 95.23 \\
\hline $\begin{array}{l}\text { Average length } \\
\theta=100, n=5\end{array}$ & 28.955 & 2.800 & 2.772 & 2.772 & 2.759 & 2.772 & 2.782 \\
\hline Coverage \% & 95.29 & 95.23 & 95.15 & 95.17 & 94.96 & 94.97 & 95.40 \\
\hline $\begin{array}{l}\text { Average length } \\
\theta=100, n=10\end{array}$ & 787.62 & 23.334 & 17.548 & 17.531 & 17.416 & 17.441 & 17.737 \\
\hline Coverage \% & 95.01 & 95.22 & 95.10 & 95.09 & 95.08 & 95.17 & 95.31 \\
\hline $\begin{array}{l}\text { Average length } \\
\theta=100, n=20\end{array}$ & 287.11 & 13.939 & 12.404 & 12.398 & 12.376 & 12.400 & 12.500 \\
\hline Coverage \% & 95.23 & 94.98 & 95.04 & 95.04 & 94.97 & 95.04 & 95.24 \\
\hline $\begin{array}{l}\text { Average length } \\
\theta=100, n=50\end{array}$ & 156.14 & 9.258 & 8.766 & 8.764 & 8.746 & 8.765 & 8.815 \\
\hline Coverage \% & 95.14 & 95.46 & 95.36 & 95.31 & 95.33 & 95.32 & 95.39 \\
\hline $\begin{array}{l}\text { Average length } \\
\theta=100, n=100\end{array}$ & 85.676 & 5.660 & 5.544 & 5.544 & 5.531 & 5.544 & 5.564 \\
\hline Coverage \% & 95.33 & 95.20 & 95.21 & 95.21 & 95.11 & 95.17 & 95.21 \\
\hline Average length & 57.889 & 3.960 & 3.921 & 3.920 & 3.911 & 3.920 & 3.930 \\
\hline
\end{tabular}

Table 2 considers the same values of $\theta$ and $n$ as Table 1 but the sample is taken from a Poisson distribution. First note that for small $\theta$ and $n$, it is possible for $x_{1}=$ $\ldots=x_{n}$ which yields $(0,0)$ using approach $1,(\bar{x}, \bar{x})$ for approach 2 and $(0,0)$ for approach 4 only when each $x_{\mathrm{i}}=0$. For these cases, this occurred less than $1 \%$ of the time and the coverage percentages and average lengths were appropriately modified counting only those cases where this phenomena did not occur. For smaller $\theta$, approaches 1 (for $\theta=1$ and 5) and 5 (for $\theta$ $=1,5$ and 10) tended to have coverage probabilities below 0.95 but these probabilities get closer to 0.95 as $\theta$ increases. Approach 4 performed the best for these values of $\theta$ followed closely by approaches 6 and 3 except when $\theta=1$ and $n=5$ or 10 where approach 6 performed the best followed by approaches 3 and 7 with approach 4 having too small covering percentages. When $\theta$ is larger $(\theta=20,50,100)$, the coverage percentages of all the approaches are all around $95 \%$ for the various $n$. In this case, approach 5 performs the best followed by approaches 4, 6 and 3; in fact, for $n$ $=20,50$ and 100, approaches 3, 4 and 6 have almost identical average lengths. Again approach 1 has much higher average lengths than the other approaches for all $\theta$ and $n$ considered.

Table 3 gives the results for various large values of $\theta$ when sampling from the Poisson distribution when $n=1$, where approaches 3 through 7 are considered. The coverage percentages of all five approaches are around $95 \%$ for all the values of $\theta$ given. The Wald approach 
(approach 4) is the dominating approach followed by the Bayesian approach assuming sampling from a Poisson population (approach 6). Approach 5 is the next best approach and approaches 3 and 5 have identical average lengths for large $\theta$. Approach 7 (Garwood) had good coverage probability but had largest average length among the five approaches. The good performance of approach 4 may be surprising as it is reasonable to suspect that it would not do well for small $n$; however, even in Table 1 and 2, approach 4 did perform well for larger $\theta$ when $n$ is small.

For Table 1 and 2, average lengths increase as $\theta$ increases for fixed $n$ for each approach and average lengths decrease as $n$ increases for fixed $\theta$ for each approach. For Table 3, average lengths increase as $\theta$ increases for each approach.

Consider comparing the results of $\left(n_{1}=1, \theta_{1}=\theta\right)$ and $\left(n_{2}=n, \theta_{2}=\theta / n\right)$ cases in Table 2 and 3 where Poisson data was assumed. For example, compare the results of the $(1,250)$ and $(50,5)$ cases or the results of the $(1,400)$ and $(20,20)$ cases. For approaches $3,4,6$ and 7 , the average length of the intervals for the $(1, \theta)$ case was about $n$ times that for the respective $(n, \theta / n)$ case. This can be seen by comparing (3) to (13), (4) to (14), (11) to (17) and (6) to (15) and noting that $Y=\sum_{i=1}^{n} X_{\mathrm{i}}$, where $X_{1}, \ldots, X_{n}$ is a random sample from a $\operatorname{Poisson}(\theta / n)$ distribution and $Y$ is a Poisson $(\theta)$ random variable. When you multiply the intervals in (3), (4) and (6) by $n$, intervals (13), (14) and (15), respectively, are obtained. Noting that the posteriors in (11) and (17) are the same when $(n, \theta / n)$ is substituted in $(11)$ and $(1, \theta)$ is substituted in (17) and when using (11), the interval for $\theta_{2}=\theta / n$ would actually be found. Unfortunately, the cases considered in Table 2 and 3 cannot be reduced due to the observation noted above only applies to approaches $3,4,6$ and 7 .

Table 2: Coverage percentages and average length of interval estimates for $\theta$ for the seven approaches for various $\theta, n$ and underlying Poisson distribution

\begin{tabular}{|c|c|c|c|c|c|c|c|}
\hline & \multicolumn{7}{|c|}{ Approach } \\
\hline & 1 & 2 & 3 & 4 & 5 & 6 & 7 \\
\hline \multicolumn{8}{|l|}{$\theta=1, n=5$} \\
\hline Coverage $\%$ & 95.43 & 95.94 & 95.91 & 87.29 & 87.06 & 95.52 & 97.87 \\
\hline Average length & 8.020 & 2.072 & 1.879 & 1.684 & 1.537 & 1.771 & 1.955 \\
\hline \multicolumn{8}{|l|}{$\theta=1, n=10$} \\
\hline Coverage $\%$ & 90.96 & 94.34 & 96.34 & 92.92 & 87.40 & 95.65 & 97.48 \\
\hline Average length & 2.866 & 1.373 & 1.285 & 1.224 & 1.056 & 1.256 & 1.342 \\
\hline \multicolumn{8}{|l|}{$\theta=1, n=20$} \\
\hline Coverage \% & 90.47 & 93.95 & 94.52 & 95.08 & 86.57 & 95.08 & 95.82 \\
\hline Average length & 1.546 & 0.916 & 0.892 & 0.871 & 0.730 & 0.882 & 0.926 \\
\hline \multicolumn{8}{|l|}{$\theta=1, n=50$} \\
\hline Coverage $\%$ & 89.75 & 94.77 & 94.54 & 95.02 & 85.15 & 95.02 & 95.47 \\
\hline Average length & 0.855 & 0.564 & 0.558 & 0.553 & 0.456 & 0.556 & 0.575 \\
\hline \multicolumn{8}{|l|}{$\theta=1, n=100$} \\
\hline Coverage $\%$ & 89.23 & 94.76 & 95.14 & 94.62 & 85.61 & 95.25 & 95.62 \\
\hline Average length & 0.578 & 0.395 & 0.393 & 0.391 & 0.321 & 0.392 & 0.402 \\
\hline \multicolumn{8}{|l|}{$\theta=5, n=5$} \\
\hline Coverage $\%$ & 95.24 & 94.57 & 94.20 & 94.63 & 92.60 & 94.63 & 95.73 \\
\hline Average length & 39.151 & 5.172 & 3.974 & 3.898 & 3.809 & 3.932 & 4.127 \\
\hline \multicolumn{8}{|l|}{$\theta=5, n=10$} \\
\hline Coverage $\%$ & 94.35 & 94.97 & 94.54 & 95.05 & 92.95 & 95.05 & 95.21 \\
\hline Average length & 14.453 & 3.122 & 2.793 & 2.766 & 2.675 & 2.780 & 2.873 \\
\hline \multicolumn{8}{|l|}{$\theta=5, n=20$} \\
\hline Coverage \% & 93.92 & 94.94 & 95.02 & 94.52 & 93.00 & 95.36 & 95.48 \\
\hline Average length & 7.746 & 2.060 & 1.967 & 1.957 & 1.879 & 1.962 & 2.011 \\
\hline \multicolumn{8}{|l|}{$\theta=5, n=50$} \\
\hline Coverage \% & 93.81 & 95.19 & 94.79 & 95.15 & 92.84 & 95.15 & 95.44 \\
\hline Average length & 4.276 & 1.264 & 1.241 & 1.239 & 1.184 & 1.240 & 1.260 \\
\hline \multicolumn{8}{|l|}{$\theta=5, n=100$} \\
\hline Coverage \% & 93.90 & 95.09 & 95.09 & 95.12 & 93.09 & 95.40 & 95.53 \\
\hline Average length & 2.888 & 0.884 & 0.877 & 0.877 & 0.837 & 0.877 & 0.887 \\
\hline \multicolumn{8}{|l|}{$\theta=10, n=5$} \\
\hline Coverage $\%$ & 95.01 & 94.94 & 94.38 & 94.59 & 93.83 & 94.41 & 95.20 \\
\hline Average length & 79.119 & 7.385 & 5.582 & 5.529 & 5.437 & 5.520 & 5.747 \\
\hline
\end{tabular}


Patricia Pepple Williamson / Journal of Mathematics and Statistics 2018, Volume 14: 219.231 DOI: 10.3844/jmssp.2018.219.231

\begin{tabular}{|c|c|c|c|c|c|c|c|}
\hline & 1 & 2 & 3 & 4 & 5 & 6 & 7 \\
\hline \multicolumn{8}{|l|}{$\theta=10, n=10$} \\
\hline Coverage \% & 94.41 & 95.04 & 95.03 & 94.68 & 94.05 & 95.33 & 95.41 \\
\hline $\begin{array}{l}\text { Average length } \\
\theta=10, n=20\end{array}$ & 28.523 & 4.390 & 3.933 & 3.914 & 3.844 & 3.922 & 4.020 \\
\hline Coverage $\%$ & 94.59 & 95.18 & 95.04 & 94.95 & 94.08 & 95.47 & 95.55 \\
\hline \multicolumn{7}{|l|}{$\theta=10, n=50$} & 2.835 \\
\hline Coverage \% & 94.59 & 95.14 & 95.01 & 95.10 & 94.13 & 95.26 & 95.25 \\
\hline $\begin{array}{l}\text { Average length } \\
\theta=10, n=100\end{array}$ & 8.566 & 1.789 & 1.755 & 1.754 & 1.714 & 1.755 & 1.774 \\
\hline Coverage \% & 94.58 & 95.24 & 95.03 & 95.29 & 94.25 & 95.29 & 95.38 \\
\hline \multicolumn{8}{|l|}{$\theta=20, n=5$} \\
\hline Coverage \% & 94.59 & 95.10 & 94.92 & 94.50 & 94.69 & 95.34 & 95.40 \\
\hline $\begin{array}{l}\text { Average length } \\
\theta=20, n=10\end{array}$ & 160.90 & 10.520 & 7.872 & 7.834 & 7.795 & 7.853 & 8.045 \\
\hline Coverage $\%$ & 95.00 & 95.25 & 95.01 & 94.79 & 94.63 & 95.36 & 95.42 \\
\hline $\begin{array}{l}\text { Average length } \\
\theta=20, n=20\end{array}$ & 57.146 & 6.127 & 5.553 & 5.540 & 5.492 & 5.547 & 5.651 \\
\hline Coverage $\%$ & 94.80 & 95.24 & 95.48 & 95.33 & 94.73 & 95.18 & 95.51 \\
\hline \multicolumn{7}{|l|}{$\theta=20, n=50$} & 3.972 \\
\hline Coverage \% & 94.48 & 95.08 & 95.04 & 95.34 & 94.69 & 95.34 & 95.36 \\
\hline $\begin{array}{l}\text { Average length } \\
\theta=20, n=100\end{array}$ & 17.069 & 2.526 & 2.480 & 2.479 & 2.450 & 2.480 & 2.499 \\
\hline Coverage $\%$ & 94.64 & 95.20 & 95.08 & 95.16 & 94.78 & 95.29 & 95.31 \\
\hline \multicolumn{8}{|l|}{$\theta=50, n=5$} \\
\hline Coverage \% & 95.10 & 95.13 & 94.96 & 95.06 & 95.04 & 95.06 & 95.45 \\
\hline $\begin{array}{l}\text { Average length } \\
\theta=50, n=10\end{array}$ & 396.95 & 16.566 & 12.418 & 12.394 & 12.369 & 12.386 & 12.581 \\
\hline Coverage \% & 95.14 & 95.03 & 94.74 & 94.86 & 94.95 & 95.11 & 95.15 \\
\hline \multicolumn{8}{|l|}{$\theta=50, n=20$} \\
\hline Coverage \% & 95.20 & 95.06 & 95.11 & 95.25 & 95.05 & 95.25 & 95.33 \\
\hline \multicolumn{8}{|l|}{$\theta=50, n=50$} \\
\hline Coverage $\%$ & 94.83 & 95.33 & 95.10 & 95.26 & 95.08 & 95.26 & 95.38 \\
\hline \multicolumn{8}{|l|}{$\theta=50, n=100$} \\
\hline Coverage \% & 94.84 & 95.22 & 95.13 & 95.14 & 95.08 & 95.20 & 95.27 \\
\hline $\begin{array}{l}\text { Average length } \\
\theta=100, n=5\end{array}$ & 28.927 & 2.799 & 2.772 & 2.772 & 2.758 & 2.772 & 2.782 \\
\hline Coverage \% & 95.10 & 95.24 & 94.99 & 94.91 & 95.02 & 95.17 & 95.46 \\
\hline $\begin{array}{l}\text { Average length } \\
\theta=100, n=10\end{array}$ & 787.59 & 23.319 & 17.545 & 17.528 & 17.510 & 17.536 & 17.741 \\
\hline Coverage \% & 94.88 & 95.19 & 94.99 & 95.15 & 95.09 & 95.15 & 95.30 \\
\hline \multicolumn{8}{|l|}{$\theta=100, n=20$} \\
\hline Coverage \% & 95.26 & 95.27 & 95.21 & 95.14 & 95.14 & 95.32 & 95.39 \\
\hline $\begin{array}{l}\text { Average length } \\
\theta=100, n=50\end{array}$ & 155.511 & 9.240 & 8.765 & 8.763 & 8.745 & 8.764 & 8.814 \\
\hline Coverage $\%$ & 95.13 & 95.41 & 95.16 & 95.14 & 95.01 & 95.22 & 95.26 \\
\hline $\begin{array}{l}\text { Average length } \\
\theta=100, n=100\end{array}$ & 85.644 & 5.660 & 5.544 & 5.544 & 5.531 & 5.544 & 5.564 \\
\hline Coverage \% & 95.04 & 95.01 & 95.13 & 95.12 & 95.03 & 95.12 & 95.18 \\
\hline Average length & 57.963 & 3.962 & 3.921 & 3.920 & 3.911 & 3.920 & 3.930 \\
\hline
\end{tabular}


Table 3: Coverage percentages and average length of interval estimates for $\theta$ for the seven approaches for $n=1$ and various $\theta$ with underlying Poisson distribution

\begin{tabular}{|c|c|c|c|c|c|c|c|}
\hline & \multicolumn{7}{|c|}{ Approach } \\
\hline & 1 & 2 & 3 & 4 & 5 & 6 & 7 \\
\hline \multicolumn{8}{|l|}{$\theta=50$} \\
\hline Coverage $\%$ & $* * * *$ & $* * * *$ & 94.83 & 95.10 & 95.10 & 95.10 & 95.46 \\
\hline $\begin{array}{l}\text { Average length } \\
\theta=100\end{array}$ & $* * * *$ & $* * * *$ & 27.929 & 27.662 & 27.912 & 27.794 & 28.760 \\
\hline Coverage $\%$ & $* * * *$ & $* * * *$ & 95.13 & 94.72 & 95.52 & 95.52 & 95.58 \\
\hline $\begin{array}{l}\text { Average length } \\
\theta=150\end{array}$ & $* * * *$ & $* * * *$ & 39.364 & 39.175 & 39.359 & 39.270 & 40.235 \\
\hline Coverage $\%$ & $* * * *$ & $* * * *$ & 95.54 & 95.46 & 95.09 & 95.09 & 95.55 \\
\hline $\begin{array}{l}\text { Average length } \\
\theta=200\end{array}$ & $* * * *$ & $* * * *$ & 48.127 & 47.973 & 48.125 & 48.051 & 49.032 \\
\hline Coverage $\%$ & $* * * *$ & $* * * *$ & 94.96 & 94.75 & 95.28 & 95.28 & 95.34 \\
\hline $\begin{array}{l}\text { Average length } \\
\theta=250\end{array}$ & $* * * *$ & $* * * *$ & 55.539 & 55.405 & 55.537 & 55.472 & 56.451 \\
\hline Coverage $\%$ & $* * * *$ & $* * * *$ & 94.85 & 95.05 & 95.05 & 95.05 & 95.52 \\
\hline $\begin{array}{l}\text { Average length } \\
\theta=300\end{array}$ & $* * * *$ & $* * * *$ & 62.076 & 61.957 & 62.075 & 62.017 & 62.986 \\
\hline Coverage $\%$ & $* * * *$ & $* * * *$ & 94.95 & 95.19 & 95.19 & 95.19 & 95.24 \\
\hline $\begin{array}{l}\text { Average length } \\
\theta=350\end{array}$ & $* * * *$ & $* * * *$ & 67.998 & 67.889 & 67.998 & 67.944 & 68.899 \\
\hline Coverage \% & $* * * *$ & $* * * *$ & 95.21 & 94.94 & 95.49 & 95.49 & 95.56 \\
\hline $\begin{array}{l}\text { Average length } \\
\theta=400\end{array}$ & $* * * *$ & $* * * *$ & 73.440 & 73.340 & 73.440 & 73.390 & 74.374 \\
\hline Coverage \% & $* * * *$ & $* * * *$ & 95.52 & 95.46 & 95.25 & 95.25 & 95.71 \\
\hline Average length & $* * * *$ & $* * * *$ & 78.491 & 78.397 & 78.491 & 78.444 & 79.424 \\
\hline
\end{tabular}

It is difficult to compare results of this simulation study with the conclusions of Schwertman and Martinez (1994), Barker (2002), Byrne and Kabaila (2005) and Patil and Kulkarni (2012) due to the various comparison criteria and values of $n$ and $\theta$ considered. However, it is fair to say that some considered the Garwood interval more favorable and the Wald interval much less favorable than this study. For instance, for $n=1$, Patil and Kulkarni (2012) determined from their numerical study that the Garwood and scores intervals had good coverage rates for $\theta$ in the interval $(0,50]$ with the Garwood interval having shortest length along with three other intervals among the 19 intervals for $\theta$ between 4 and 50 . Furthermore, they recommended to avoid the Wald interval due to low coverage rates for all $\theta$ they considered. Tanusit (2012) considered $\theta=1,2, \ldots, 5$ and $n=10,11, \ldots, 100$ and recommended the scores and Wald intervals for small $n$ and large $n$, respectively.

The adaptation of the Wald interval given in Khamkong (2012) is referred to as AWC and is given by

$$
\bar{X}+\mathrm{z}_{\alpha / 2}^{2} /(2 n) \pm z_{\alpha / 2} \sqrt{\bar{X} / n}
$$

The simulation study considered $\theta=1,1.5,3,5$ and 10 and $n=15,25,50$ and 100; hence, there is some overlap with the numerical study of this paper. Note that the length of AWC equals the length of the Wald interval given in (4) and the length of AWC is less than the length of the scores interval given in (3). From the simulation of Khamkong, the coverage rates of the scores interval and AWC are usually equal and below $95 \%$. Requiring a coverage rate of at least $95 \%$, the table below using Poisson data notes the approach that yielded the shortest length when AWC is compared to those in this paper for $\theta$ and $n$ that include or are somewhat close to values in the simulation of Khamkong. Referring to the AWC interval as approach 8 , the Wald interval and the second Bayes approach dominate for these values of $\theta$ and $n$.

\begin{tabular}{|c|c|c|c|c|c|c|c|c|c|}
\hline \multirow{3}{*}{$\begin{array}{l}n \\
\theta\end{array}$} & \multicolumn{3}{|c|}{20} & \multicolumn{3}{|c|}{50} & \multicolumn{3}{|c|}{100} \\
\hline & & & ----- & & -1 & 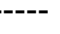 & & & \\
\hline & 1 & 5 & 10 & 1 & 5 & 10 & 1 & 5 & 10 \\
\hline Best approach & 4 & 6 & 6 & 4 & 4 & 4,8 & 6 & 4 & 4,8 \\
\hline
\end{tabular}

Assuming the $\mathrm{N}(\theta, \theta)$ model, note that $T=\sum_{i=1}^{n} X_{i}^{2}$ is a minimal sufficient statistic for $\theta$ because the joint density of $X_{1}, \ldots, X_{n}$ in (7) can be written as:

$$
\begin{aligned}
& \mathrm{f}(x \mid \theta) \\
& =(2 \pi \theta)^{-n / 2} \exp \{-n \theta / 2\} \exp \left\{\sum_{i=1}^{n} x_{i}\right\} \exp \left\{-\left(\sum_{i=1}^{n} x_{i}^{2}\right) /(2 \theta)\right\} .
\end{aligned}
$$


By the Sufficiency Principle, any inference about $\theta$ should depend on the sample $\left(X_{1}, \ldots, X_{n}\right)$ only through $T$. Note that $Q_{1}$ and $Q_{2}$ involve $\bar{X}$ as well as $T$ and $Q_{3}$ does not involve $T$. Assuming the Poisson $(\theta)$ model, the Wald interval based on $Q_{4}$, the Bayesian approach yielding (12) and the exact Garwood interval have $\sum_{i=1}^{n} X_{i}$ as a minimal sufficient statistic for $\theta$. The only approach that solely utilizes $T$ is the Bayesian approach that assumes the $\mathrm{N}(\theta, \theta)$ model. Hence, it makes sense that approach 5 is the dominating approach for normal data as in Table 1 .

\section{Example}

After the National Basketball Association (NBA) and American Basketball Association (ABA) merged in 1976, the San Antonio Spurs have won five NBA championships and 22 division titles. For the 1979-1980 season, the NBA adopted the three-point shot, where a player's feet must be completely behind the three-point line at the time of the shot or jump in order to make a three-point attempt. In recent years, the Spurs have typically been a very good three-point shooting team.
As an example, consider the number of three-point attempts by the San Antonio Spurs during the 20112012, 2012-2013 and 2013-2014 regular seasons where they were in the top three in team three point shooting percentage. There are 82 games in a regular season; however, the 2011-2012 season consisted of only 66 regular season games because it started late due to a lockout. For the 230 regular season games in the time period of interest, the actual average number of threepoint attempts by the Spurs was 21.4 per game. Assuming the number of three-point attempts in a game has a Poisson $(\theta)$ distribution, consider estimating $\theta$ based on a random sample of size 20 from the 230 games of interest. Using $\mathrm{R}$ to select the random sample, the games selected were on February 18, March 28, April 9 and 18, November 10 and 17 of 2012, February 19 and 22, March 3, 16 and 27, November 11 and December 31 of 2013, January 4 and 17, February 10, March 16 and 28, April 3 and 14 of 2014 which yielded three-point attempts of 28 , $20,24,21,18,27,30,22,20,17,15,28,20,18,20,17$, 16, 24, 22 and 17, respectively. This sample resulted in a sample mean of 21.200 and a sample variance of 19.221 which are fairly close; hence, the Poisson model seems feasible. The interval estimates of $\theta$ for Approaches 1 through 7 are given in the table below.

\begin{tabular}{lllllll}
\hline & Approach & & & & & \\
& & & & & & \\
& 1 & 2 & 3 & 4 & 5 & 7 \\
\hline Interval lower bound & 11.116 & 19.148 & 19.276 & 19.182 & 19.185 & 19.206 \\
Interval upper bound & 41.004 & 23.252 & 23.316 & 23.218 & 23.177 & 23.244 \\
Length of interval & 29.888 & 4.104 & 4.040 & 4.036 & 3.992 & 4.038 \\
\hline
\end{tabular}

From the table, note that the actual value of $\theta, 21.4$, is in each interval. Approach 1 yielded by far the widest interval while approach 5 (first Bayes) yielded the shortest interval followed closely by approaches 4 (Wald), 6 (second Bayes) and 3 (scores). This is what is expected considering our discussion of Table 2 for $\theta=20=n$.

Of interest may also be the number of three-pointers made for these 20 games in the sample; these values were $12,10,7,8,7,16,10,4,9,6,10,13,9,4,8,5,10$,
13,9 and 4, where the actual mean number of threepointers made in a game was $\theta=8.3$. This sample resulted in a mean and variance of 8.700 and 10.642 , respectively. From the table below, note that 8.3 lies within each interval. Approach 1 is again the worst approach, whereas, approach 5 is the best followed closely by approaches 4,6 and 3 . From our discussion of Table 2 when $\theta$ is small and $n=20$, approach 4 was expected to perform the best.

\begin{tabular}{|c|c|c|c|c|c|c|c|}
\hline & \multicolumn{7}{|c|}{ Approach } \\
\hline & 1 & 2 & 3 & 4 & 5 & 6 & 7 \\
\hline Interval lower bound & 6.155 & 7.173 & 7.500 & 7.407 & 7.554 & 7.430 & 7.455 \\
\hline Interval upper bound & 22.702 & 10.227 & 10.092 & 9.993 & 10.094 & 10.020 & 10.093 \\
\hline Length of interval & 16.547 & 3.054 & 2.592 & 2.586 & 2.540 & 2.590 & 2.638 \\
\hline
\end{tabular}

\section{Conclusion}

When sampling from a $\mathrm{N}(\theta, \theta)$ or a Poisson $(\theta)$ distribution, the Bayesian credible interval obtained assuming normal data (approach 5) appears to perform very well for various values of $\theta$ and $n$. The only exceptions to this occurs when sampling from a $\mathrm{N}(\theta, \theta)$ distribution and $\theta$ and $n$ are small where approach 3 (scores) is preferred and when sampling from a $\operatorname{Poisson}(\theta)$ distribution when $\theta$ is small or 
when $\theta$ is not small and $n=1$ where approach 4 (Wald) is preferred.

In statistics, it is always desired to improve on statistical methods in various inference problems. Estimating a Poisson mean is not a new problem and has been discussed in the literature for years. Although the distribution was named after Simeon Denis Poisson in the 1800's, some believe it should have been named after Abraham de Moivre who actually appears to be the first to discover it in the early 1700 's.

\section{Acknowledgement}

The author would like to thank Professor Wojbor Woyczynski of Case Western Reserve University and the reviewers for their helpful comments and suggestions.

\section{Ethics}

The author declares that there is no conflict of interest regarding the publication of this manuscript.

\section{References}

Barker, L., 2002. A comparison of nine confidence intervals for a Poisson parameter when the expected number of events $\leq 5$. Am. Stat., 56: 85-89. DOI: $10.1198 / 000313002317572736$

Byrne, J. and P. Kabaila, 2005. Comparison of Poisson confidence intervals. Commun. Stat. Theory Meth., 34: 545-556. DOI: 10.1081/STA-200052109

Casella, G. and R.L. Berger, 2002. Statistical Inference. 2nd Edn., Thompson Learning, Pacific Grove, ISBN-10: 0495391875, pp: 660.
Garwood, F., 1936. Fiducial limits for the Poisson distribution. Biometrika, 28: 437-442.

DOI: $10.2307 / 2333958$

Jeffreys, S.H., 1961. Theory of Probability. 3rd Edn., Clarendon Press, Oxford, pp: 447.

Kabaila, P. and J. Byrne, 2001. Exact short Poisson confidence intervals. Canad. J. Stat., 29: 99-106. DOI: $10.2307 / 3316053$

Khamkong, M., 2012. Approximate confidence interval for the mean of a Poisson distribution. Open J. Stat., 2: 204-207. DOI: 10.4236/ojs.2012.22024

Mood, A., F. Graybill and D. Boes, 1974. Introduction to the Theory of Statistics. 3rd Edn., McGraw-Hill, ISBN-10: 0070854653, pp: 564.

Patil, V.V. and H.V. Kulkarni, 2012. Comparison of confidence intervals for the Poisson mean: Some new aspects. Stat. J., 10: 211-227. https://www.ine.pt/revstat/pdf/rs120203.pdf

Sahai, H. and A. Khurshid, 1993. Confidence intervals for the mean of a Poisson distribution: A review. Biometrical J., 7: 857-867. DOI: $10.1002 /$ bimj.4710350716

Schwertman, N.C. and R.A. Martinez, 1994. Approximate Poisson confidence limits. Commun. Stat. Theory Meth., 23: 1507-1529. DOI: $10.1080 / 03610929408831336$

Tanusit, M., 2012. Two-sided confidence intervals for the Poisson means. Int. J. Model. Optimiz., 2: 589-591. DOI: 10.7763/IJMO.2012.V2.189 